\title{
Splitting with Different Growth Rates for Linear Discrete-time Skew-evolution Semiflows in Banach Spaces
}

\author{
Diana Borlea ${ }^{12}$
}

\begin{abstract}
In this paper we intend to study three concepts of $(h, k)$-splitting for skew-evolution semiflows, which model discretetime variational systems in Banach spaces. We also aim to give connections between them, emphasized by counterexamples and we propose an open problem.
\end{abstract}

AMS Subject Classification (2000). 34D09; 34D05.

Keywords. discrete-time skew-evolution semiflow, $(h, k)$ splitting, strong $(h, k)$-splitting, weak $(h, k)$-splitting.

\section{Introduction}

There has been an extraordinary development in the theory of asymptotic properties regarding the evolution operators. Results concerning the asymptotic behaviors of dynamical systems were published firstly in [17], whereas further points of view might be found in [11]. Some extensions in the infinite dimensional case are illustrated in [8], [16] and in [18] and for the

\footnotetext{
${ }^{1}$ Faculty of Mathematics and Computer Science, West University of Timişoara, Romania

${ }^{2}$ Faculty of Social Science, Humanities and Physical Education and Sports, "Vasile Goldiş" Western University of Arad, Romania, dianab268@yahoo.com
} 
case of discrete-linear systems, the works [1], [3] and [4] are worth of mentioning, where the theory of difference equations was further developed and interesting new results were obtained. Other various and relevant concepts of dichotomy are presented and analyzed in [2], [5], [6], [7], [14], [19] and [20]. The term of skew-evolution semiflow, which was first mentioned in [13], should be studied in the nonuniform case, as it relies on three variables. Only two of these variables make the generalization possible for skew-product semiflows and evolution operators. The study of asymptotic behaviors for skew-evolution semiflows in the nonuniform setting appears to be normal and connected to the other third variable. The term is also relevant to the stability theory both from a uniform and nonuniform point of view and it has been mentioned by some researchers, such as P. Viet Hai (see [9] and [10]) and T. Yue, X. Q. Song and D. Q. Li (see [23]). Their studies have revealed newer concepts of skew-evolution semiflows and led to further aspects of asymptotic behaviors and applications. A part of these characteristics of skew-evolution semiflows are depicted in [21], [22] and [23]. As an additional remark, we can also state that some recent results in studying the exponential splitting property for evolution operators were successfully pointed out in [15].

Three concepts of $(h, k)$-splitting for skew-evolution semiflows which model discrete-time variational systems in Banach spaces are going to be analyzed in the present paper. We will also exemplify different types of splitting properties, by providing definitions and counterexamples, making connections and last but not least, pointing out an open problem which this study addresses, which concerns the implication between the uniformly strong $(h, k)$-splitting and the strong $(h, k)$-splitting concepts.

\section{Preliminaries}

Let $(X, d)$ be a metric space, $V$ a Banach space and $\mathcal{B}(V)$ the Banach space of all bounded linear operators acting on $V$. We denote by

$$
\Delta=\left\{(m, n) \in \mathbb{N}_{+}^{2}: m \geq n\right\} .
$$

Definition 2.1. A mapping $\varphi: \Delta \times X \rightarrow X$ is called a discrete evolution semiflow on $X$ if the following conditions hold:

$\left(\right.$ des $\left._{1}\right) \varphi(m, m, x)=x$, for all $(m, x) \in \mathbb{N} \times X$

$\left(\right.$ des $\left._{2}\right) \varphi(m, n, \varphi(n, p, x))=\varphi(m, p, x)$, for all $(m, n),(n, p) \in \Delta, x \in X$. 
Definition 2.2. A mapping $\Phi: \Delta \times X \rightarrow \mathcal{B}(V)$ is called a discrete evolution cocycle over the evolution semiflow $\varphi$ if:

$\left(\right.$ dec $\left._{1}\right) \Phi(m, m, x)=I$, for all $m \in \mathbb{N}, x \in X$ (where $I: V \rightarrow V$ - is the identity operator on $V$ ).

$\left(d_{e} c_{2}\right) \Phi(m, n, \varphi(n, p, x)) \Phi(n, p, x)=\Phi(m, p, x)$, for all $(m, n),(n, p) \in \Delta$ and for all $x \in X$.

If $\Phi$ is a discrete evolution cocycle over the discrete evolution semiflow $\varphi$, then the pair $C=(\varphi, \Phi)$, defined by:

$$
C: \Delta \times X \times V \rightarrow X \times V, C(m, n, x, v)=(\varphi(m, n, x), \Phi(m, n, x) v)
$$

is called a discrete skew-evolution semiflow on $X \times V$.

Definition 2.3. A sequence $h: \mathbb{N} \rightarrow[1,+\infty)$ is said to be a growth rate if $h$ is nondecreasing and $\lim _{n \rightarrow \infty} h(n)=+\infty$.

Note. In what follows, $h, k: \mathbb{N} \rightarrow[1,+\infty)$ will denote two arbitrary growth rates and they will be used in several examples.

\section{Sequences of projections on Banach spaces and two leading examples}

Definition 3.1. An operator valued mapping $P: \mathbb{N} \times X \rightarrow \mathcal{B}(V)$ is called a sequence of projections if

$$
P(n, x) P(n, x)=P(n, x), \quad \text { for all }(n, x) \in \mathbb{N} \times X .
$$

If $P: \mathbb{N} \times X \rightarrow \mathcal{B}(V)$ is a sequence of projections, then the sequence $Q: \mathbb{N} \times X \rightarrow \mathcal{B}(V)$ defined by $Q(n, x)=I-P(n, x)$ is also a sequence of projections, called the complementary sequence of projections of $P: \mathbb{N} \times X \rightarrow \mathcal{B}(V)$.

Definition 3.2. Given $N: \mathbb{N} \rightarrow[1,+\infty)$ a growth rate, we say that the sequence of projections $P: \mathbb{N} \times X \rightarrow \mathcal{B}(V)$ is $N$-bounded if there exist $\gamma \geq 0$ such that

$$
\|P(n, x)\| \leq N(n)^{\gamma}, \quad \text { for all }(n, x) \in \mathbb{N} \times X .
$$

If $\gamma=0$ then we say that the sequence $P: \mathbb{N} \times X \rightarrow \mathcal{B}(V)$ is bounded. 
Below, two examples of sequences of projections will be presented, with the aid of which several constructions of discrete linear systems will be made in order to delimit the presented concepts from this paper.

Example 3.1. Consider the Banach space

$$
V=l^{\infty}(\mathbb{N}, \mathbb{R})=\left\{v: \mathbb{N} \rightarrow \mathbb{R}: \sup _{j \in \mathbb{N}}|v(j)|<+\infty\right\}
$$

endowed with the norm

$$
\|v\|_{\infty}=\sup _{j \in \mathbb{N}}|v(j)| .
$$

We define $P: \mathbb{N} \times X \rightarrow \mathcal{B}(V)$ by

$$
P(n, x) v=v \cdot \chi_{3 \mathbb{N}} \quad \text { for all }(n, x, v) \in \mathbb{N} \times X \times V,
$$

where $\chi_{A}$ is the characteristic function of the set $A \subset \mathbb{N}$.

We have that $P: \mathbb{N} \times X \rightarrow \mathcal{B}(V)$ is a sequence of projections with its complementary given, for all $(n, x, v) \in \mathbb{N} \times X \times V$, by

$$
Q(n, x) v=\left(\chi_{3 \mathbb{N}+1}+\chi_{3 \mathbb{N}+2}\right) \cdot v .
$$

Moreover, we have that

$$
\|P(n, x)\|=\|Q(n, x)\|=1, \quad \text { for all }(n, x) \in \mathbb{N} \times X .
$$

Example 3.2. We will denote by $\mathbb{O}$ the set of nonnegative odd numbers and by $\mathbb{E}$ the set of nonnegative even numbers. Consider $p: \mathbb{N} \rightarrow[1, \infty), p(n)=p_{n}$, to be a nondecreasing sequence. Consider the Banach space

$$
V=l^{2}(\mathbb{N}, \mathbb{R})=\left\{v: \mathbb{N} \rightarrow \mathbb{R}: \sum_{j=0}^{\infty}|v(j)|^{2}<\infty\right\}
$$

endowed with the norm

$$
\|v\|_{2}=\left(\sum_{j=0}^{\infty}|v(j)|^{2}\right)^{\frac{1}{2}}
$$

Let $P: \mathbb{N} \times X \rightarrow \mathcal{B}(V)$ be defined by

$$
P(n, x) v(j)=\left(v(j)+p_{n} v(j+1)\right) \cdot \chi_{\mathbb{E}}(j), \quad \text { for all } n, j \in \mathbb{N}, v \in V, x \in X .
$$

For $n \in \mathbb{N}, x \in X$ and $v \in V$ we denote by

$$
u_{n}=P(n, x) v
$$


It follows that for all $j \in \mathbb{N}$ the following relations hold:

$$
\left\{\begin{array}{l}
u_{n}(2 j)=v(2 j)+p_{n} v(2 j+1) \\
u_{n}(2 j+1)=0
\end{array}\right.
$$

Hence

$$
P(n, x) u_{n}(j)=\left(u_{n}(j)+p_{n} u_{n}(j+1)\right) \cdot \chi_{\mathbb{E}}(j)=\left\{\begin{array}{l}
u_{n}(j), \quad j=2 l \\
0, \quad j=2 l+1
\end{array}\right.
$$

from where we deduce that

$$
P(n, x) u_{n}=u_{n}
$$

which shows us that $P(n, x)$ is a projection on $V$.

In addition, for every $n \in \mathbb{N}, x \in X$ and $v \in V$ the following estimations hold:

$$
\begin{aligned}
\|P(n, x) v\|_{2} & =\left(\sum_{j=0}^{\infty}\left|u_{n}(j)\right|^{2}\right)^{\frac{1}{2}}=\left(\sum_{j=0}^{\infty}\left|u_{n}(2 j)\right|^{2}\right)^{\frac{1}{2}} \\
& =\left(\sum_{j=0}^{\infty}\left|v(2 j)+p_{n} v(2 j+1)\right|^{2}\right)^{\frac{1}{2}} \leq \\
& \leq\left(1+p_{n}\right)\|v\|_{2}
\end{aligned}
$$

which implies the following upper-bound estimation for the family of projections $P: \mathbb{N} \times X \rightarrow \mathcal{B}(V)$ :

$$
\|P(n, x)\| \leq 1+p_{n}, \quad \text { for all }(n, x) \in \mathbb{N} \times X .
$$

Consider now the sequence $v_{0} \in V$ given by

$$
v_{0}(j)=\frac{1}{j+1} \cdot \chi_{\mathbb{E}}(j), \quad j \in \mathbb{N} .
$$

We have that

$$
\begin{aligned}
\left\|P(n, x) v_{0}\right\|_{2} & =\left(\sum_{j=0}^{\infty}\left|v_{0}(j)+p_{n} v_{0}(j+1)\right|^{2} \chi_{\mathbb{E}}(j)\right)^{\frac{1}{2}}= \\
& =\left(\sum_{j=0}^{\infty}\left|v_{0}(2 j)+p_{n} v_{0}(2 j+1)\right|^{2}\right)^{\frac{1}{2}}= \\
& =\left(\sum_{j=0}^{\infty} p_{n}^{2} \frac{1}{(2 j+1)^{2}}\right)^{\frac{1}{2}}=p_{n}\left\|v_{0}\right\|_{2}
\end{aligned}
$$


from where we deduce the following double inequality:

$$
p_{n} \leq\|P(n, x)\| \leq 1+p_{n}, \quad \text { for all }(n, x) \in \mathbb{N} \times X .
$$

The complementary sequence of $P$ is given by

$$
Q(n, x) v(j)=-p_{n} v(j+1) \chi_{\mathbb{E}}(j)+v(j) \chi_{\mathbb{E}}(j), \quad(x, v, n, j) \in X \times V \times \mathbb{N}^{2} .
$$

We observe that if $(m, n) \in \Delta, x \in X$ and $v \in V$ the following estimations hold:

$$
\begin{aligned}
\|Q(n, x) v\|_{2}^{2} & =\sum_{j=0}^{\infty}|v(j)|^{2}=\sum_{j=0}^{\infty}\left|p_{n} v(2 j+1)\right|^{2}+\sum_{j=0}^{\infty}|v(2 j+1)|^{2}= \\
& =\left(1+p_{n}^{2}\right) \sum_{j=0}^{\infty}|v(2 j+1)|^{2}
\end{aligned}
$$

from where we deduce that

$$
\begin{aligned}
\|Q(n, x) v\|_{2} & =\left(1+p_{n}^{2}\right)^{\frac{1}{2}}\left(\sum_{j=0}^{\infty}|v(2 j+1)|^{2}\right)^{\frac{1}{2}} \leq \\
& \leq\left(1+p_{m}^{2}\right)^{\frac{1}{2}}\left(\sum_{j=0}^{\infty}|v(2 j+1)|^{2}\right)^{\frac{1}{2}}=\|Q(m, x) v\|_{2} .
\end{aligned}
$$

Consider now $m, n \in \mathbb{N}, x \in X$ and $v \in V$. We denote by

$$
u_{n}=P(n, x) v \quad \text { and } \quad v_{n}=Q(n, x) v .
$$

It follows that

$$
v(2 j)=-p_{n} x(2 j+1) \quad \text { and } \quad v(2 j+1)=x(2 j+1), \quad \text { for all } j \in \mathbb{N} .
$$

From the definition of $P$, we have that

$$
\begin{aligned}
P(m, x) P(n, x) v(j) & =P(m, x) u_{n}(j)=\left(u_{n}(j)+p_{m} u_{n}(j+1)\right) \chi_{\mathbb{E}}(j)= \\
& =u_{n}(j) \chi_{\mathbb{E}}(j)=u_{n}(j) \\
Q(m, x) Q(n, x) v(j) & =Q(m, x) v_{n}(j)=-p_{m} v_{n}(j+1) \chi_{\mathbb{E}}(j)+v_{n}(j) \chi_{\mathbb{E}}(j)= \\
& =-p_{m} v(j+1) \chi_{\mathbb{E}}(j)+v(j) \chi_{\mathbb{E}}(j)=Q(m, x) v(j) \\
Q(m, x) P(n, x) v(j) & =Q(m, x) u_{n}(j)=-p_{m} u_{n}(j+1) \chi_{\mathbb{E}}(j)+u_{n}(j) \chi_{\mathbb{E}}(j)=0 .
\end{aligned}
$$

We conclude that for all $(m, n, x) \in \mathbb{N}^{2} \times X$ the following relations hold:

$$
\begin{aligned}
& P(m, x) P(n, x)=P(n, x), \\
& Q(m, x) Q(n, x)=Q(m, x), \\
& Q(m, x) P(n, x)=0 .
\end{aligned}
$$




\section{Concepts of $(h, k)$-splitting for linear discrete-time skew-evolution semiflows}

If $C$ is a discrete skew-evolution semiflow on $X \times V$ and $P: \mathbb{N} \times X \rightarrow \mathcal{B}(V)$ is a sequence of projections, then we will say that $(C, P)$ is a splitting pair.

Definition 4.1. Let $P: \mathbb{N} \times X \rightarrow \mathcal{B}(V)$ be a sequence of projections. We say that the splitting pair $(C, P)$ admits a $(h, k)$-splitting and we denote $(h, k)-$ $s$ if there exist a nondecreasing sequence $N: \mathbb{N} \rightarrow[1, \infty)$ and constants $\alpha, \beta \in \mathbb{R}$ with $\alpha<\beta$ and $\gamma \geq 0$ such that for all $(m, n, x) \in \Delta \times X$ the following conditions hold:

$\left(h s_{1}\right) h(n)^{\alpha}\|\Phi(m, n, x) P(n, x)\| \leq N(n)^{\gamma} h(m)^{\alpha}$

$\left(k s_{2}\right) k(m)^{\beta} \leq N(n)^{\gamma} k(n)^{\beta}\|\Phi(m, n, x) Q(n, x)\|$.

If $\gamma=0$ then we say that the pair $(C, P)$ admits a uniformly $(h, k)$-splitting and we denote $u-(h, k)-s$.

If in the above definition, we have that $\alpha<0<\beta$, then we say that $(C, P)$ admits a $(h, k)$-dichotomy.

An example of a discrete linear system admitting a $(h, k)$-splitting is given in Example 4.1.

Example 4.1. (A splitting pair which admits a (uniform) $(h, k)$-splitting) Consider the Banach space

$$
V=L^{2}((1, \infty), \mathbb{R}) \oplus l^{\infty}(\mathbb{N}, \mathbb{R})
$$

endowed with the norm

$$
\|v\|=\max \left\{\|f\|_{2},\|s\|_{\infty}\right\}, \quad v=(f, s) \in V .
$$

Define, for every $(m, n, x) \in \Delta \times X$,

$$
\Phi(m, n, x)(f, s)=\left(f_{m}^{n}, s_{m}^{n}\right)
$$

where

$$
f_{m}^{n}(\tau)=f\left(\left(\frac{h(n)}{h(m)}\right)^{2} \cdot \tau\right), \quad \tau \in(1, \infty)
$$

and

$$
s_{m}^{n}(j)=\frac{k(n)}{k(m)} \cdot 1, \quad j \in \mathbb{N} .
$$


Let $P: \mathbb{N} \times X \rightarrow \mathcal{B}(V)$ be defined by

$$
P(n, x)(f, s)=(f, 0), \quad \text { for all }(f, s) \in V, \quad(n, x) \in \mathbb{N} \times X .
$$

Then $(C, P)$ is a splitting pair. Let $(f, s) \in X$.

$$
\begin{aligned}
\|\Phi(m, n, x) P(n, x)(f, s)\| & =\|\Phi(m, n, x)(f, 0)\|=\left\|f_{m}^{n}\right\|_{2}= \\
& =\left(\int_{1}^{\infty}\left|f\left(\left(\frac{h(n)}{h(m)}\right)^{2} \cdot \tau\right)\right|^{2} d \tau\right)^{\frac{1}{2}}= \\
& =\left(\int_{\left(\frac{h(m)}{h(n)}\right)^{2}}^{\infty}|f(\xi)|^{2} \cdot\left(\frac{h(n)}{h(m)}\right)^{4} d \xi\right)^{\frac{1}{2}} \leq \\
& \leq\left(\frac{h(n)}{h(m)}\right)^{2}\|f\|_{2}= \\
& =\left(\frac{h(n)}{h(m)}\right)^{2}\|P(n, x)(f, s)\| ; \\
\|\Phi(m, n, x) Q(n, x)(f, s)\| & =\|\Phi(m, n, x)(0, s)\|=\left\|s_{m}^{n}\right\|_{\infty}= \\
& =\frac{k(n)}{k(m)}\|Q(n, x)(f, s)\|_{\infty}
\end{aligned}
$$

from where we obtain that the pair $(C, P)$ admits a (uniform) $(h, k)-s$ with $N \equiv 1, \alpha=-2$ and $\beta=-1$.

Remark 4.2. If $(C, P)$ admits a $(h, k)$-dichotomy, then it follows that $(C, P)$ admits a $(h, k)$-splitting. The converse is not generally true. Indeed, Example 4.2 proves this fact, by taking into account that $\beta<0$. The same is true for the associated uniform concepts, which is pointed out by the fact that in Example 4.1, again, $\beta$ is not strictly positive.

Proposition 4.3. If $(C, P)$ admits $a(h, k)-s$ with constants $N \geq 1$ and $\gamma \geq 0$ given by Definition 4.1, then

$$
\max \{\|P(n, x)\|,\|Q(n, x)\|\} \leq 2 N(n)^{\gamma}, \quad \text { for all }(n, x) \in \mathbb{N} \times X \text {. }
$$

Proof. It follows from $\left(h s_{1}\right)$ by taking $m=n$ and from the fact that

$$
\|Q(n, x)\| \leq 2\|P(n, x)\| .
$$


Corollary 4.4. If $(C, P)$ admits a $u-(h, k)-s$ then $P: \mathbb{N} \times X \rightarrow \mathcal{B}(V)$ is bounded.

Remark 4.5. If the pair $(C, P)$ admits a $u-(h, k)-s$ then it also admits a $(h, k)-s$. The converse is not generally true, fact illustrated by Example 4.2 .

Example 4.2. On the Banach space $V=l^{2}(\mathbb{N}, \mathbb{R})$, consider the sequence of projections given by Example 3.2. By taking $p_{n}=N(n)$, with $N: \mathbb{N} \rightarrow$ $[1, \infty)$ begin an arbitrary growth rate (in order to have infinite limit) and

$\Phi(m, n, x)=\left(\frac{h(m)}{h(n)}\right)^{-2} P(n, x)+\frac{k(n)}{k(m)} Q(m, x), \quad$ for all $(m, n, x) \in \Delta \times X$.

Taking into account $(3.4)$, we observe that $(C, P)$ is a splitting pair, with

$$
\begin{aligned}
& \Phi(m, n, x) P(n, x)=\left(\frac{h(m)}{h(n)}\right)^{-2} P(n, x) \quad \text { and } \\
& \Phi(m, n, x) Q(n, x)=\frac{k(n)}{k(m)} Q(m, x),
\end{aligned}
$$

for all $(m, n, x) \in \Delta \times X$. It follows that for all $(m, n, x) \in \Delta \times X$,

$$
\|\Phi(m, n, x) P(n, x)\|=\left(\frac{h(m)}{h(n)}\right)^{-2}\|P(n, x)\| \leq\left(\frac{h(m)}{h(n)}\right)^{-2} \cdot N(n)
$$

which implies that

$$
h(n)^{-2}\|\Phi(m, n, x) P(n, x)\| \leq h(m)^{-2} N(n)
$$

and

$N(n)\|\Phi(m, n, x) Q(n, x)\| \geq\|\Phi(m, n, x) Q(n, x)\|=\frac{k(n)}{k(m)}\|Q(m, x)\| \geq \frac{k(n)}{k(m)}$,

which shows us that $(C, P)$ admits a $(h, k)-s$, with $N=\gamma=1, \alpha=-2$ and $\beta=-1$. Having in mind that

$$
\sup _{n \in \mathbb{N}}\|P(n, x)\|=+\infty
$$

by Corollary 4.4, we conclude that $(C, P)$ does not admit a $u-(h, k)-s$.

Definition 4.6. Let $P: \mathbb{N} \times X \rightarrow \mathcal{B}(V)$ be a sequence of projections. We say that the pair $(C, P)$ admits a strong $(h, k)$-splitting and we denote $s-(h, k)-s$ if there exist a nondecreasing sequence $N: \mathbb{N} \rightarrow[1, \infty), \alpha, \beta \in \mathbb{R}$ with $\alpha<\beta$ and $\gamma \geq 0$ such that for all $(m, n, x, v) \in \Delta \times X \times V$ the following properties hold: 
$\left(s h s_{1}\right) h(n)^{\alpha}\|\Phi(m, n, x) P(n, x) v\| \leq N(n)^{\gamma} h(m)^{\alpha}\|P(n, x) v\| ;$

$\left(s k s_{2}\right) k(m)^{\beta}\|Q(n, x) v\| \leq N(n)^{\gamma} k(n)^{\beta}\|\Phi(m, n, x) Q(n, x) v\|$.

If $\gamma=0$ then we say that the pair $(C, P)$ admits a uniformly strong $(h, k)$ splitting and we denote $u . s-(h, k)-s$.

If in the above definition, we have that $\alpha<0<\beta$, then we say that $(C, P)$ admits a strong $(h, k)$-dichotomy.

An example of a system admitting a strong $(h, k)$-splitting is given in Example 4.1.

Remark 4.7. If $(C, P)$ admits a strong $(h, k)$-dichotomy, then it follows that $(C, P)$ admits a strong $(h, k)$-splitting. The converse is not generally true.

Remark 4.8. If the pair $(C, P)$ admits a strong $(h, k)$-splitting, then for all $(m, n, x) \in \Delta \times X$ the following implication is true:

$$
\Phi(m, n, x) Q(n, x) v=0 \Rightarrow Q(n, x) v=0 .
$$

The following remarks point out some connections between the behaviors described above.

Remark 4.9. If the pair $(C, P)$ admits a u.s $-(h, k)-s$, then it also admits a $s-(h, k)-s$. The converse is momentarily an open problem.

Remark 4.10. If the pair $(C, P)$ admits a $s-(h, k)-s$, it does not imply that it also admits a $(h, k)-s$. The following holds for the concepts of $u . s-(h, k)-s$ and $u-(h, k)-s$. In order to justify these facts, we point out Example 4.3.

Example 4.3. Let $V=l^{2}(\mathbb{N}, \mathbb{R}), N: \mathbb{N} \rightarrow[1, \infty)$ an arbitrary growh rate and as in Example 4.2, take

$$
\Phi(m, n, x)=\left(\frac{h(m)}{h(n)}\right)^{-2} P(n, x)+\frac{k(n)}{k(m)} Q(m, x), \quad(m, n, x) \in \Delta \times X
$$

and

$$
p_{n}=e^{N(n)}, \quad n \in \mathbb{N} .
$$

From (4.1) we deduce that $(C, P)$ admits a $u . s-(h, k)-s$, hence it also admits a $s-(h, k)-s$. If we assume that the pair $(C, P)$ admits a $(h, k)-s$ then, by Proposition 4.3, we would obtain that there exist $N: \mathbb{N} \rightarrow[1, \infty)$ a growth rate and $\gamma \geq 0$ such that

$$
\|P(n, x)\| \leq N(n)^{\gamma}, \quad \text { for all }(n, x) \in \mathbb{N} \times X .
$$


Having in mind the choice of the sequence $p$ and (3.2), we obtain the contradiction

$$
e^{N(n)}=p_{n} \leq\|P(n, x)\| \leq N(n)^{\gamma}, \quad \text { for all }(n, x) \in \mathbb{N} \times X .
$$

Hence $(C, P)$ is not $(h, k)-s$ therefore it is also not $u-(h, k)-s$.

Remark 4.11. If the pair $(C, P)$ admits a $(h, k)-s$, it does not necessarily follows that it admits a $s-(h, k)-s$. The same holds for the concepts of $u-(h, k)-s$ and $u . s-(h, k)-s$. In order to justify these facts, we point out Example 4.4.

Example 4.4. On $V=l^{\infty}(\mathbb{N}, \mathbb{R})$ let $P: \mathbb{N} \times X \rightarrow \mathcal{B}(V)$ the sequence of projections defined in Example 3.1. We define, for $(m, n, x) \in \Delta \times X$ and $(v, j) \in V \times \mathbb{N}$ we have that

$\Phi(m, n, x) v(j)= \begin{cases}\left(\frac{h(m)}{h(n)}\right)^{2} v(j) \chi_{3 \mathbb{N}}(j)+\left(\frac{k(m)}{k(n)}\right)^{3} v(j) \chi_{3 \mathbb{N}+1}(j), & \text { if } m \geq n+1 \\ v(j), & \text { if } m=n,\end{cases}$

where $3 \mathbb{N}+k=\{3 n+k: n \in \mathbb{N}\} \subset \mathbb{N}$, for each $k \in \mathbb{N}$.

Then $(C, P)$ is a splitting pair with the property that for all $(m, n, x, v) \in$ $\Delta \times X \times V$ and $j \in \mathbb{N}$ the following representations hold:

$$
\Phi(m, n, x) P(n, x) v(j)=\left(\frac{h(m)}{h(n)}\right)^{2} v(j) \chi_{3 \mathbb{N}}(j)
$$

and

$$
\Phi(m, n, x) Q(n, x) v(j)= \begin{cases}\left(\frac{k(m)}{k(n)}\right)^{3} v(j) \chi_{3 \mathbb{N}+1}(j), & \text { if } m \geq n+1 \\ v(j)\left(\chi_{3 \mathbb{N}+1}(j)+\chi_{3 \mathbb{N}+2}(j)\right), & \text { if } m=n\end{cases}
$$

It follows that

$\|\Phi(m, n, x) P(n, x) v\|_{\infty}=\left(\frac{h(m)}{h(n)}\right)^{2} \sup _{j \geq 0}\left|v(j) \chi_{3 \mathbb{N}}(j)\right|=\left(\frac{h(m)}{h(n)}\right)^{2}\|P(n, x) v\|_{\infty}$

from where

$$
\|\Phi(m, n, x) P(n, x)\| \leq\left(\frac{h(m)}{h(n)}\right)^{2} .
$$

If $m \geq n+1$, let $v_{1} \in V$ given by

$$
v_{1}(j)=\frac{1}{j+1} \chi_{3 \mathbb{N}+1}(j), \quad j \in \mathbb{N} .
$$


Then

$$
\left\|\Phi(m, n, x) Q(n, x) v_{1}\right\|_{\infty}=\left(\frac{k(m)}{k(n)}\right)^{3}\left\|Q(n, x) \chi_{3 \mathbb{N}+1} v_{1}\right\|_{\infty}=\left(\frac{k(m)}{k(n)}\right)^{3}\left\|v_{1}\right\|_{\infty}
$$

from where

$$
\left(\frac{k(m)}{k(n)}\right)^{3} \leq\|\Phi(m, n, x) Q(n, x)\| .
$$

The case $m=n$ is obvious. Hence $(C, P)$ admits a $u-(h, k)-s$, hence it is also $(h, k)-s$.

Assume by a contradiction that the pair $(C, P)$ admits a $s-(h, k)-s$. Let $(1,0, x) \in \Delta \times X$. Obviously, the sequence

$$
v_{2}: \mathbb{N} \rightarrow \mathbb{R}, \quad v_{2}(j)=\frac{1}{j+1} \chi_{3 \mathbb{N}+2}(j), \quad j \in \mathbb{N}
$$

belongs to $l^{\infty}(\mathbb{N}, \mathbb{R})$. We have that

$$
Q(0, x) v_{2}=v_{2} \neq 0
$$

and for all $j \in \mathbb{N}$,

$$
\Phi(1,0, x) Q(0, x) v_{2}(j)=2 v_{2}(j) \chi_{3 \mathbb{N}+1}(j)=\frac{2}{j+1} \chi_{3 \mathbb{N}+2}(j) \cdot \chi_{3 \mathbb{N}+1}(j)=0 .
$$

By Remark 4.8, we obtain that $(C, P)$ cannot admit a $s-(h, k)-s$, neither a $u . s-(h, k)-s$.

Definition 4.12. Let $P: \mathbb{N} \times X \rightarrow \mathcal{B}(V)$ be a sequence of projections. We say that the pair $(C, P)$ admits a weak $(h, k)$-splitting and we denote $w-(h, k)-s$ if there exist a nondecreasing sequence $N: \mathbb{N} \rightarrow[1, \infty)$ and constants $\alpha, \beta \in \mathbb{R}$ with $\alpha<\beta$ and $\gamma \geq 0$ such that for all $(m, n, x) \in \Delta \times X$ the following hold:

$$
\begin{aligned}
& \left(w h s_{1}\right) \quad h(n)^{\alpha}\|\Phi(m, n, x) P(n, x)\| \leq N(n)^{\gamma} h(m)^{\alpha}\|P(n, x)\| ; \\
& \left(w k s_{2}\right) \quad k(m)^{\beta}\|Q(n, x)\| \leq N(n)^{\gamma} k(n)^{\beta}\|\Phi(m, n, x) Q(n, x)\| .
\end{aligned}
$$

If $\gamma=0$ then we say that the pair $(C, P)$ admits uniformly weak $(h, k)$ splitting and we denote $u . w-(h, k)-s$.

If in the above definition, we have that $\alpha<0<\beta$, then we say that $(C, P)$ admits a weak $(h, k)$-dichotomy. 
Remark 4.13. If $(C, P)$ admits a weak $(h, k)$-dichotomy, then it admits a weak $(h, k)$-splitting. The converse is not generally true. Indeed, from Proposition 4.16, we have that the splitting pair admits a weak $(h, k)$-splitting, with $\alpha=2$ hence it cannot admit a weak $(h, k)$-dichotomy.

Remark 4.14. Consider $(C, P)$ to be a splitting pair. The following assertions hold true:

(i) If $(C, P)$ admits a $u . w-(h, k)-s$ then it also admits a $w-(h, k)-s$.

(ii) If the pair $(C, P)$ admits a $s-(h, k)-s$ then it also admits a $w-(h, k)-s$. Moreover, if $(C, P)$ admits a u.s $-(h, k)-s$ then it also admits a $u . w-(h, k)-s$.

(iii) If the pair $(C, P)$ admits a $(h, k)-s$ then it also admits a $w-(h, k)-s$.

Indeed, below we justify the above assertions.

(i) The necessity is obvious. The fact that the converse doesn't generally hold, for the moment, remains an open problem. We do not own an example of a splitting pair $(C, P)$ which is $w-(h, k)-s$, but is not $u . w-(h, k)-s$.

(ii) It is obvious, by passing to the operator norm in Definition 4.6.

(iii) Let $N: \mathbb{N} \rightarrow[1, \infty), \alpha, \beta \in \mathbb{R}$ with $\alpha<\beta$ and $\gamma \geq 0$ given by Definition 4.6. By Proposition 4.3, we have that

$$
1 \leq \max \{\|P(n, x)\|,\|Q(n, x)\|\} \leq 2 N(n)^{\gamma}, \quad \text { for all }(n, x) \in \mathbb{N} \times X .
$$

Let $(m, n) \in \Delta$. From the fact that

$$
h(n)^{\alpha}\|\Phi(m, n, x) P(n, x)\| \leq N(n)^{\gamma} h(m)^{\alpha} \leq 2 N^{2}(n)^{2 \gamma} h(m)^{\alpha}\|P(n, x)\|
$$

and

$$
\begin{aligned}
k(m)^{\beta}\|Q(n, x)\| & \leq N(n)^{\gamma} k(n)^{\beta}\|\Phi(m, n, x) Q(n, x)\| \cdot\|Q(n, x)\| \leq \\
& \leq 2 N^{2}(n)^{2 \gamma} k(n)^{\beta}\|\Phi(m, n, x) Q(n, x)\|,
\end{aligned}
$$

it follows that $(C, P)$ admits a $w-(h, k)-s$.

Taking into account the facts from the above proposition, (iii), we can state the following result.

Corollary 4.15. If the pair $(C, P)$ admits a $u-(h, k)-s$ then it also admits a u.w $-(h, k)-s$. 
More connections are shown in the following proposition.

Proposition 4.16. For a given splitting pair $(C, P)$, the following assertions hold:

(i) If the pair $(C, P)$ admits a $w-(h, k)-s$, it does not necessarily follow that it admits a $(h, k)-s$.

(ii) If the pair $(C, P)$ admits a $w-(h, k)-s$, it does not necessarily follow that it admits a $s-(h, k)-s$.

Proof. The arguments that sustain the above assertions are presented below.

(i) Indeed, by considering the splitting pair $(C, P)$ from Example 4.3, we have that it admits a u.s - $(h, k)-s$, hence $u . w-(h, k)-s$ which implies the fact that it is also $w-(h, k)-s$. From the facts proven in the same example, we have that $(C, P)$ cannot admit a $(h, k)-s$, neither $u-(h, k)-s$.

(ii) By considering the splitting pair from Example 4.4 and taking into account that

$$
\|P(n, x)\|=\|Q(n, x)\|=1
$$

for all $(n, x) \in \mathbb{N} \times X$, it follows that for all $(m, n, x) \in \Delta \times X$,

$$
\|\Phi(m, n, x) P(n, x)\| \leq\left(\frac{h(m)}{h(n)}\right)^{2}
$$

and

$$
\left(\frac{k(m)}{k(n)}\right)^{3}\|Q(n, x)\| \leq\|\Phi(m, n, x) Q(n, x)\| .
$$

It follows that $(C, P)$ admits a $u . w-(h, k)-s$ (with $N=1$ as a constant sequence, $\alpha=2<\beta=3)$, hence $w-(h, k)-s$. From the assertions proven in Example 4.4, we have that $(C, P)$ is not $s-(h, k)-s$ hence neither is it $u . s-(h, k)-s$.

Remark 4.17. The connections between the concepts studied in this paper is given by the following diagram:

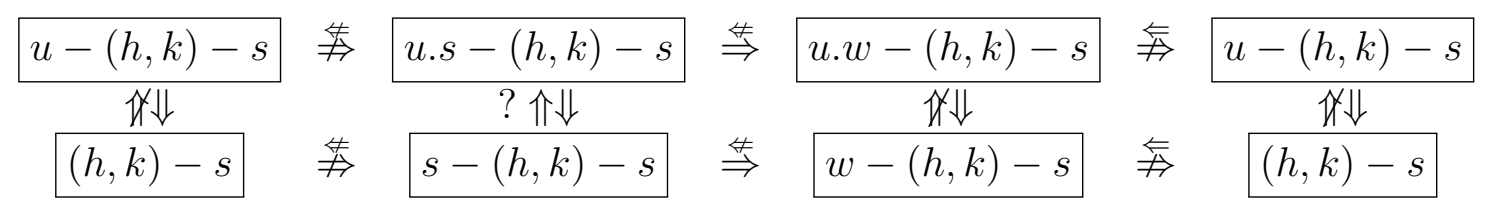




\section{References}

[1] R. P. Agarwal, Difference equations and inequalities: theory, methods and applications, Marcel Dekker, Second edition, 2000

[2] V. Lakshmikantham J. Appell N. van Minh and P. Zabreiko, A general model of evolutionary processes. Exponential dichotomy - I, II, Nonlinear Anal., 21, (1993), 207-218, 219-225

[3] B. Aulbach and J. Kalbrenner, Exponential forward splitting for noninvertible difference equations, Comp. Math. Appl., 42, (2001), 743-751

[4] B. Aulbach and S. Siegmund, The dichotomy spectrum for noninvertible systems of linear difference equations, J. Difference Equ. Appl., 7, (2001), 895-913

[5] M.-G. Babuţia and M. Megan, Exponential dichotomy concepts for evolution operators on the half-line, Ann. Acad. Rom. Sci. Ser. Math. Appl., 7, (2015), 209226

[6] S. N. Chow and H. Leiva, Existence and roughness of the exponential dichotomy for linear skew-product semiflows in Banach spaces, J. Differential Equations, 120, (1995), 429-477

[7] W. A. Coppel, Dichotomies in stability theory, Lecture Notes in Math., 629, (1978)

[8] J. L. Daleckii and M. G. Krĕ̌n, Stability of Solutions of Differential Equations in Banach Spaces, Translations of Mathematical Monographs, 43, (1974)

[9] P. Viet Hai, Continuous, discrete characterizations for the uniform exponential stability of linear skew-evolution semiflows, Nonlinear Anal., 72, (2010), 4390-4396

[10] P. Viet Hai, Discrete, continuous versions of Barbashin-type theorem of linear skewevolution semiflows, Appl. Anal., 90, (2011), 1897-1907

[11] J. L. Massera and J. J. Schäffer, Linear Differential Equations, Function Spaces, Pure Appl. Math., 21, (1966)

[12] M. Megan and I.-L. Popa, Exponential splitting for nonautonomous linear discrete-time systems in Banach spaces, J. Comput. Appl. Math., 312, (2017), 181191

[13] M. Megan and C. Stoica, Exponential instability of skew-evolution semiflows in Banach spaces, Studia Univ. Babeş-Bolyai Math., 53, (2008), 17-24

[14] M. Megan and C. Stoica, Concepts of dichotomy for skew-evolution semiflows in Banach spaces, Ann. Acad. Rom. Sci. Ser. Math. Appl., 2, (2010), 125-140

[15] C. S. Stoica and M. Megan C. L. Mihit, On uniform exponential splitting for noninvertible evolution operators in Banach spaces, An. Univ. Vest. Timişoara, Ser. Mat.-Inf. LIII, 2, (2015), 121-131

[16] A. Pazy, Semigroups of Linear Operators, Applications to Partial Differential Equations, Springer Verlag, New York, 1983

[17] O. Perron, Die Stabitätsfrage bei Differentialgleichungen, Math. Z., 32, (1930), 703-728 
[18] R. J. Sacker and G. R. Sell, Dichotomies for linear evolutionary equations in Banach spaces, J. Differential Equations, 113, (1994), 17-67

[19] B. Sasu and A. L. Sasu, Exponential dichotomy, $\left(l^{p}, l^{q}\right)$-admissibility on the halfline, J. Math. Anal. Appl., 316, (2006), 397-408

[20] C. Stoica and D. Borlea, On $H$-dichotomy for skew-evolution semiflows in Banach spaces, Theory, Applications of Mathematics $\mathcal{E}$ Computer Science, 2, (2012), 29-36

[21] C. Stoica and D. Borlea, Exponential stability versus polynomial stability for skewevolution semiflows in infinite dimensional spaces, Theory, Applications of Mathematics Computer \&3 Science, 4, (2014), 221-229

[22] C. Stoica and M. Megan, On uniform exponential stability for skew-evolution semiflows on Banach space, Nonlinear Anal., 72, (2010), 1305-1313

[23] X. Q. Song and D. Q. Li T. Yue, On weak exponential expansiveness of skewevolution semiflows in Banach spaces, J. Inequal. Appl., (2014), 1-6

Received: 22.08 .2017

Accepted: 22.12.2017

Revised: 21.12 .2017 